\title{
Performance comparison of gel and capillary electrophoresis-based microsatellite genotyping strategies in a population research and kinship testing framework
}

\author{
Julissa J. Sánchez-Velásquez, Lorenzo E. Reyes-Flores, Carmen Yzásiga-Barrera and Eliana Zelada-Mázmela* (i)
}

\begin{abstract}
Objective: The advancement of molecular techniques in an era in which high-throughput sequencing has revolutionized biology renders old-fashioned alternatives to high-throughput methods obsolete. Such advanced molecular techniques, however, are not yet accessible to economically disadvantaged region-based laboratories that still obtain DNA profiles using gel-based techniques. To explore whether cost-efficient techniques can produce results that are as robust as those obtained using high-throughput methods, we compared the performance of polyacrylamide gel electrophoresis (PAGE)- and capillary electrophoresis (CE)-derived genomic data in estimating genetic diversity and inferring relatedness using 70 individuals of fine flounder (Paralichthys adspersus) selected from a hatchery population and genotyped for five microsatellite loci.

Results: Here, we show that PAGE- and CE-derived genomic datasets yield comparable genetic diversity levels regarding allelic diversity measures and heterozygosity. However, relatedness inferred from each dataset showed that the categorization of dyads in the different relationship categories strongly differed. This suggests that while scientists can reliably use PAGE-derived genomic data to estimate genetic diversity, they cannot use the same for parentage testing. The findings could help laboratories committed to population research not be discouraged from using the PAGE system if high-throughput technologies are unavailable and the method is adequate to address the biological question.
\end{abstract}

Keywords: Capillary electrophoresis, Genetic diversity, Polyacrylamide gel electrophoresis, Relatedness

\section{Introduction}

Ever since their discovery in 1981 [1], the usefulness of microsatellites, due to their hypervariability and ubiquitous occurrence, has been astounding to geneticists [2]. Microsatellites are short tandem repeats of almost anything from 1 to around 6 bp [2]. They are highly informative, codominant, and transferable among

\footnotetext{
*Correspondence: ezelada@uns.edu.pe

Laboratory of Genetics, Physiology, and Reproduction, Faculty

of Sciences, Universidad Nacional del Santa, Av Universitaria s/n,

02712 Nuevo Chimbote, Peru
}

phylogenetically-related species [3]. As such, microsatellites are powerful tools commonly used in population research to infer genetic diversity, genetic structure, and mating systems [4-6]. Microsatellites have also found their application in linkage-disequilibrium, in which associations between markers and traits are searched, and hitchhiking mapping, in which genome-wide surveys are used to identify regions showing positive selection $[7,8]$. However, to obtain reliable information, microsatellite genotyping should be performed with extreme accuracy since high error rates could inject bias into the 
downstream analysis and, therefore, alter ecological and evolutionary conclusions $[9,10]$.

Besides allelic dropouts and null alleles, the most common errors during microsatellite genotyping include contaminant DNA, incorrect data entry, and scoring errors [11]. Although most of these errors can be detected if the genotypification is repeated, the proportion of misscored alleles, which can be up to $80 \%$, will depend on the researchers' genotyping method [12]. The gold standard for microsatellite genotyping is capillary electrophoresis $(\mathrm{CE})$, a technology that accurately scores the alleles owing to its technology that implements automated allele-call programs [13]. However, because this method requires sophisticated instruments, it is usually impractical for many laboratories in countries having no access to cutting-edge technologies $[14,15]$. Nonetheless, more costly-effective methods such as polyacrylamide gel electrophoresis (PAGE) have been remarkably resilient in competition against sequencing techniques [16], and are still being utilized by researchers, especially in population research [17-19]; though the accuracy of their results compared to those obtained using high-throughput (HTP) methods is still arguable [12, 20-22].

Here, we present a direct comparison between PAGE and $\mathrm{CE}$ methods by applying them in the genotypification of five microsatellite loci in an economically important fish population. To the best of our knowledge, this study is the first of its kind that compares not only allele frequency data but also the different genetic diversity parameters analyzed in population research. Moreover, this study evaluates the performance of the PAGE method by comparing the results of relatedness analysis to those obtained using the CE method.

\section{Main text}

\section{Materials and methods}

\section{DNA extraction and PCR amplification}

Total 70 adults of Paralichthys adspersus (Steindachner, 1867) belonging to a hatchery population were genotyped by PAGE and CE methods using the flanking primers of five microsatellite loci developed for $P$. olivaceus (Temminck \& Schlegel, 1846): Poli9TUF, Poli28TUF [23], Po35, Po91 [24], and KOP45 [25]. DNA was extracted from approximately $20 \mathrm{mg}$ of caudal fin tissue using the SDS-proteinase K/phenol-chloroform digestion method adapted from Taggart et al. [26]. The PCR reactions were performed in $7 \mu \mathrm{L}$ reaction mixture containing $4.32 \mu \mathrm{L}$ PCR water, $0.66 \mu \mathrm{L}$ Taq Buffer KCl-MgCl2 (10X), $0.51 \mu \mathrm{L}$ $\mathrm{MgCl}_{2}(25 \mathrm{mM}), 0.33 \mu \mathrm{L}$ dNTPs $(2.5 \mathrm{mM}), 0.06 \mu \mathrm{L}$ forward/reverse primer (12.5 $\mu \mathrm{M}$ each), $0.06 \mu \mathrm{L}$ Maximo Taq DNA Polymerase (5 U/ $\mu \mathrm{L})$ (GeneON, Deutschland, Germany), and $1 \mu \mathrm{L}$ DNA $(25 \mathrm{ng} / \mu \mathrm{L})$. Thermal cycling conditions for each locus are detailed in Additional file 1: Table S1.

\section{PAGE}

PCR amplification products were visualized on $12 \%$ polyacrylamide gels following the standard Laemmli system for discontinuous gel electrophoresis [27]. Gels containing $12 \%$ separating gel and $4 \%$ stacking gel were prepared from a stock solution of $40 \%$ Acrylamide/Bis Solution 19:1 (Bio-Rad, California, USA). The separating gel contained $2667 \mu \mathrm{L}$ distilled water, $1800 \mu \mathrm{L}$ of $40 \%$ Acrylamide/Bis solution, and $1500 \mu \mathrm{L}$ Tris- $\mathrm{HCl}(1.5 \mathrm{M}$, $\mathrm{pH} 8.8) .7 \mathrm{~cm}$ separating gels were prepared into a plate sandwich of a total length of $10 \mathrm{~cm}$ and with a $1-\mathrm{mm}$ thick spacer. The gels were polymerized chemically by the addition of $3 \mu \mathrm{L}$ tetramethylethylenediamine (TEMED) and $30 \mu \mathrm{L}$ ammonium persulfate (APS) (10\%). The stacking gel contained $1906.38 \mu \mathrm{L}$ distilled water, $300 \mu \mathrm{L}$ of $40 \%$ Acrylamide/Bis solution, and $760.62 \mu \mathrm{L}$ Tris- $\mathrm{HCl}$ $(0.5 \mathrm{M}, \mathrm{pH} 6.8)$, and was polymerized in the same way as for separating gel. After gel polymerization, the plate sandwich was placed into the Ommi PAGE CVS10D gel system (Cleaver Scientific Ltd., Warwickshire, UK). Samples were prepared using $6 \mu \mathrm{L}$ of PCR amplification product and $1 \mu \mathrm{L}$ of $6 \mathrm{X}$ DNA loading dye (Thermo Scientific, Massachusetts, USA). Electrophoresis was carried out with a voltage of $85 \mathrm{~V}$ for about $2.5 \mathrm{~h}$. DNA bands were revealed using a silver nitrate staining adapted from Rangel-Villalobos and colleagues [28]. Allele sizes were estimated in comparison with a 300 bp ladder (Thermo Scientific) using Quantity One ${ }^{\circledR}$ 1-D Analysis software (Bio-Rad).

CE

PCR amplification products were also genotyped by means of automated parallel $\mathrm{CE}$ using the Fragment Analyzer $^{\mathrm{TM}}$ Automated CE System (Agilent, California, USA). Separation gel and samples were prepared using the DNF-900 dsDNA Reagent Kit (Agilent) following the manufacturer's protocol. Allele scoring was performed using PROSize data analysis software (Agilent) by interpolating their position to a 35-500 bp DNA marker and 75-400 bp range DNA ladder (Agilent).

\section{Genetic diversity}

Typographic errors, i.e., the misinterpretation of allele banding patterns because of stutter bands, and allelic dropout were determined using Micro-Checker v.2.2.3 [29]. The frequency of null alleles was calculated using ML-Null Freq v.1.0 [30]. The polymorphic information content (PIC) at each locus was calculated using CERVUS v.3.0.7 [31]. The diversity for each locus was quantified as the number of alleles per locus (A), the effective 
number of alleles $\left(a_{\mathrm{e}}\right)$, allelic frequency, observed heterozygosity (Ho), and expected heterozygosity $(\mathrm{He})$ using GenAlEx v.6.5 [32]. Allelic richness (R) was evaluated using HP-Rare v.1.0 [33]. Statistical differences between the genetic diversity measures obtained from the PAGEand CE-derived genomic datasets were subsequently tested using unpaired Student's t tests on GraphPad Prism v.7.0. The obtained $P$ values $<0.05$ were considered to be statistically significant. Further data visualization was performed in R [34].

\section{Relatedness estimation}

The coefficient of relatedness $\left(r_{x y}\right)$ was estimated using the method-of-moment estimator developed by Wang $\left(r_{w}\right)$ [35] through Coancestry v.1.2.1 [36]. The cutoff value to group the formed dyads in the different relationship categories, full-sibs (FS), half-sibs (HS), and unrelated (UR), was established as the midpoint between the arithmetic mean $r_{w}$ values of any two adjacent distributions, as suggested by Blouin et al. [37, 38]. Following Blouin et al. suggestion, FH would be the dyads with $r_{w}$ values between 0.5 and 0.375 , HF the dyads with $r_{w}$ values between 0.375 and 0.125 , and UR the dyads with an $r_{w}$ lower than 0.125. It has to be noted, however, that although the $r_{w}$ was developed from the identical by descent (IBD)-based concept of relatedness, where $r_{x y}$ can only go from 0 to 1 if neither of the two individuals being compared is inbred, this method-of-moment estimator provide negative values if the average relatedness among sampled individuals becomes close to zero $[39,40]$. This occurs because to calculate $r_{x y}$, the allele frequency data is estimated from the current sample instead of an ancestral population (as assumed when the estimators were developed) [39]. Negative values, however, have biological meaning if they are understood as the correlation of homologous genes between and within individuals due to shared ancestry as conceived by Wright $[39,41]$ in the original correlation concept of relatedness. Thus, if negative values are found, they indicate the individuals being compared are less related in ancestry than the average [39]; they belong to the UR category.

\section{Results}

\section{Genetic diversity}

Except for Poli9TUF, the number of alleles detected by PAGE and CE methods varied depending on the specific locus (Additional file 1: Table S2). Particularly in eight samples, the CE method was more sensitive and able to separate PCR products that differed by only $2 \mathrm{bp}$. The total number of alleles in both derived datasets ranged from 86 to 90 . The genetic diversity estimated from both genomic datasets was also not significantly different (in all cases $P>0.05$ ) (Fig. 1a-f). The PAGE-derived genomic data gave a mean PIC value of $0.85 \pm 0.008$. The average $\mathrm{A}, \mathrm{a}_{\mathrm{e}}$, and $\mathrm{R}$ were evaluated as $17.20 \pm 0.859$, $8.77 \pm 0.431$, and $14.03 \pm 0.661$. The average value of $\mathrm{He}$ was $0.87 \pm 0.007$, while that of Ho was $0.42 \pm 0.021$. Similarly, using $\mathrm{CE}$-derived genomic data, we inferred the mean PIC value as $0.85 \pm 0.008$. The average $\mathrm{A}, \mathrm{a}_{\mathrm{e}}$, and $\mathrm{R}$ were $18.00 \pm 0.956,8.97 \pm 0.532$, and $16.13 \pm 0.739$. The average value of $\mathrm{He}$ was $0.87 \pm 0.007$, while that of $\mathrm{Ho}$ was $0.43 \pm 0.018$. The frequency of null alleles obtained from both datasets also showed no significant differences $(P=0.726)$ (Fig. $1 \mathrm{~g})$. The allele frequency spectra obtained by both methods were also comparable, except for Po35 and KOP45 (Fig. 1h).

\section{Patterns of relatedness}

The average values for the $r_{w}$ estimator inferred from the PAGE and CE datasets supported the category unrelated as the most probable type of relationship between the individuals from the captive population of $P$. adspersus, with average values ranging from -0.111 to -0.093 . However, strong differences in the categorization of HS and FS were observed (Fig. 2a, b). For HS, only $22.76 \%$ of the dyads identified as such using the CE-derived dataset coincided with the dyads identified as HS using the PAGE dataset, while both datasets shared no dyad in the FS category. Instead, out of the total dyads identified as FS using the CE-derived dataset, we categorized $36.36 \%$ as HS and $63.64 \%$ as UR using the PAGE-derived dataset.

To validate our analyses, we also determined genetic diversity and relatedness from 40 wild individuals of $P$. adspersus by using the PAGE and CE genotyping methods. The results were similar to those obtained using the captive population: no difference in genetic diversity but great differences in kinship assignment were found (Additional file 1: Figs. S1, S2). Therefore, while microsatellite allelic data obtained from the PAGE system seem reliable to determine genetic diversity, the same seems unreliable to infer relatedness compared to that obtained using the CE method.

\section{Discussion}

Increasing the studies that can face the ongoing biodiversity and climate crisis in countries without access to cutting-edge technologies will need that the relevance of their studies be valued for their biological significance rather than the fanciness of the technique utilized [15]. However, the analytical and methodological limitations of the allegedly old-fashioned methods and their adequateness to address the biological question asked need to be considered. In this study, we demonstrated that the PAGE- and CE-derived genomic data yield nearly identical genetic diversity levels for a captive population of $P$. adspersus. Compared to the CE 

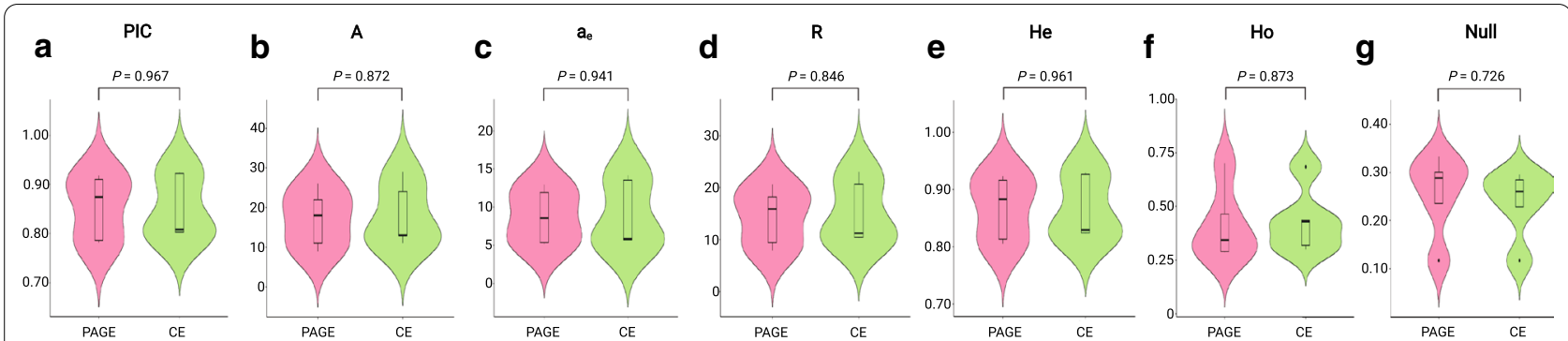

h
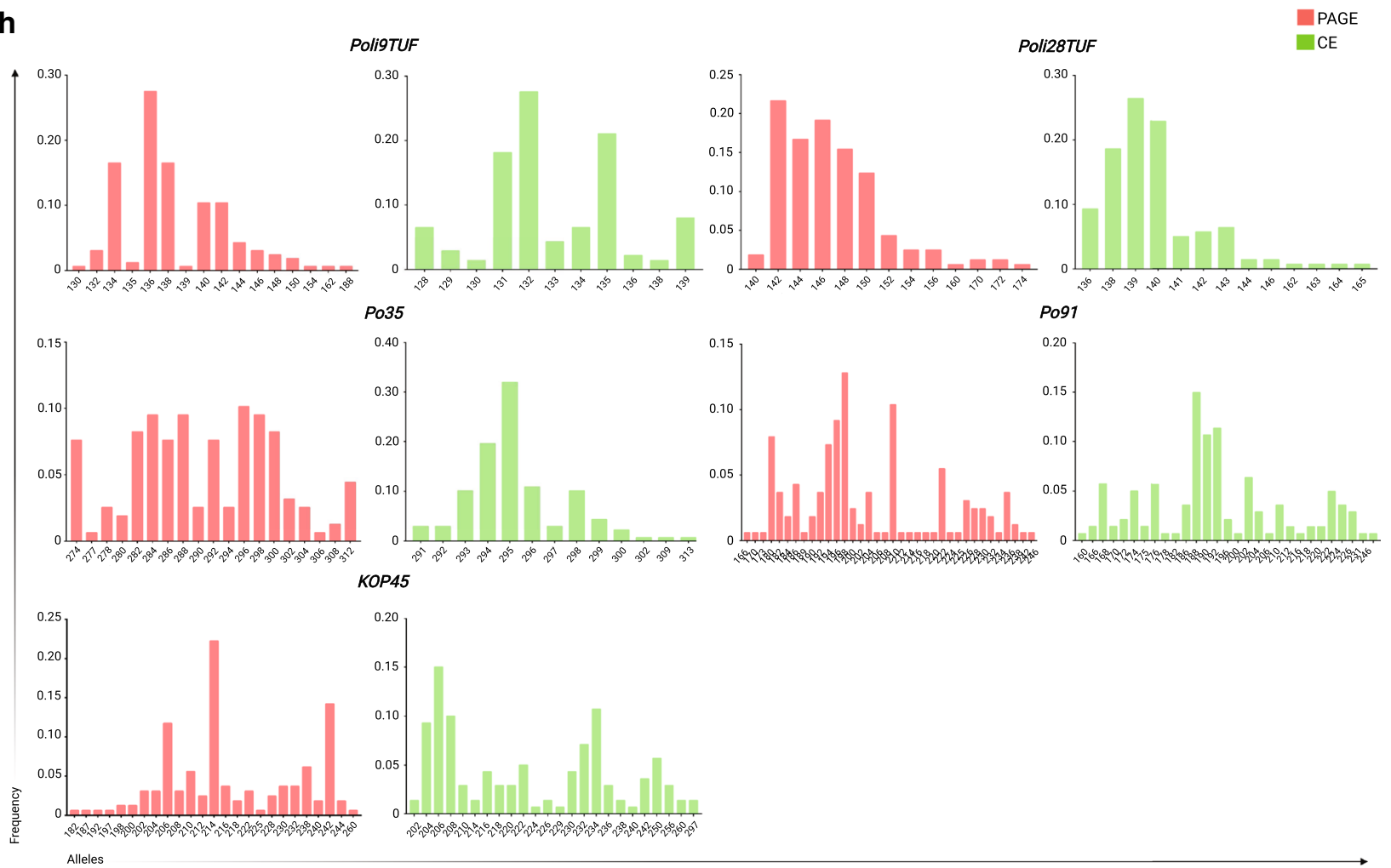

Fig. 1 Polyacrylamide gel electrophoresis (PAGE)- and capillary electrophoresis (CE)-derived genomic data give comparable genetic diversity measures. Total 70 samples of a captive population of fine flounder (Paralichthys adspersus) were genotyped for five microsatellite loci using PAGE and CE methods, and the genetic diversity levels obtained from both genomic datasets were compared. a-f Violin plots with included boxplots summarizing the data distribution of different genetic diversity parameters. $\mathbf{g}$ Violin plots with included boxplots summarizing the distribution of null allele frequencies. Each box plot shows the median (middle line) and interquartile range (boxes). The bottom and top of each box indicate the 25th and 75th percentiles, while whiskers represent the minimum and maximum. $\mathbf{h}$ Allele frequency spectra of the five microsatellite loci used. Statistical analysis was performed using unpaired t tests. PIC polymorphic information content; $A$ number of alleles; $a_{e}$ effective number of alleles; $R$ allelic richness; Ho observed heterozygosity; He expected heterozygosity

method, however, the PAGE method failed to provide similar allele frequency spectra in two out of the five microsatellite loci used, a phenomenon mainly attributed to the misinterpretation of allele banding patterns $[12,20]$. Since two previous studies with the PAGE method have provided allele frequency data that agree with that obtained using the CE method, by as much as $99 \%[16,21]$, methodological issues in this study can also not be ruled out as an error source. These findings highlight the limitations of non-HTP methods for accurate allele scoring and present avenues for improved error reduction by adjusting the data collection process. For example, Pagel et al. [21] indicate that to increase the power of resolution and avoid doubtful alleles, the PAGE's electrophoretic conditions can be improved by decreasing the voltage and increasing the gel concentration and running time, conditions that even though make the protocols more time consuming 


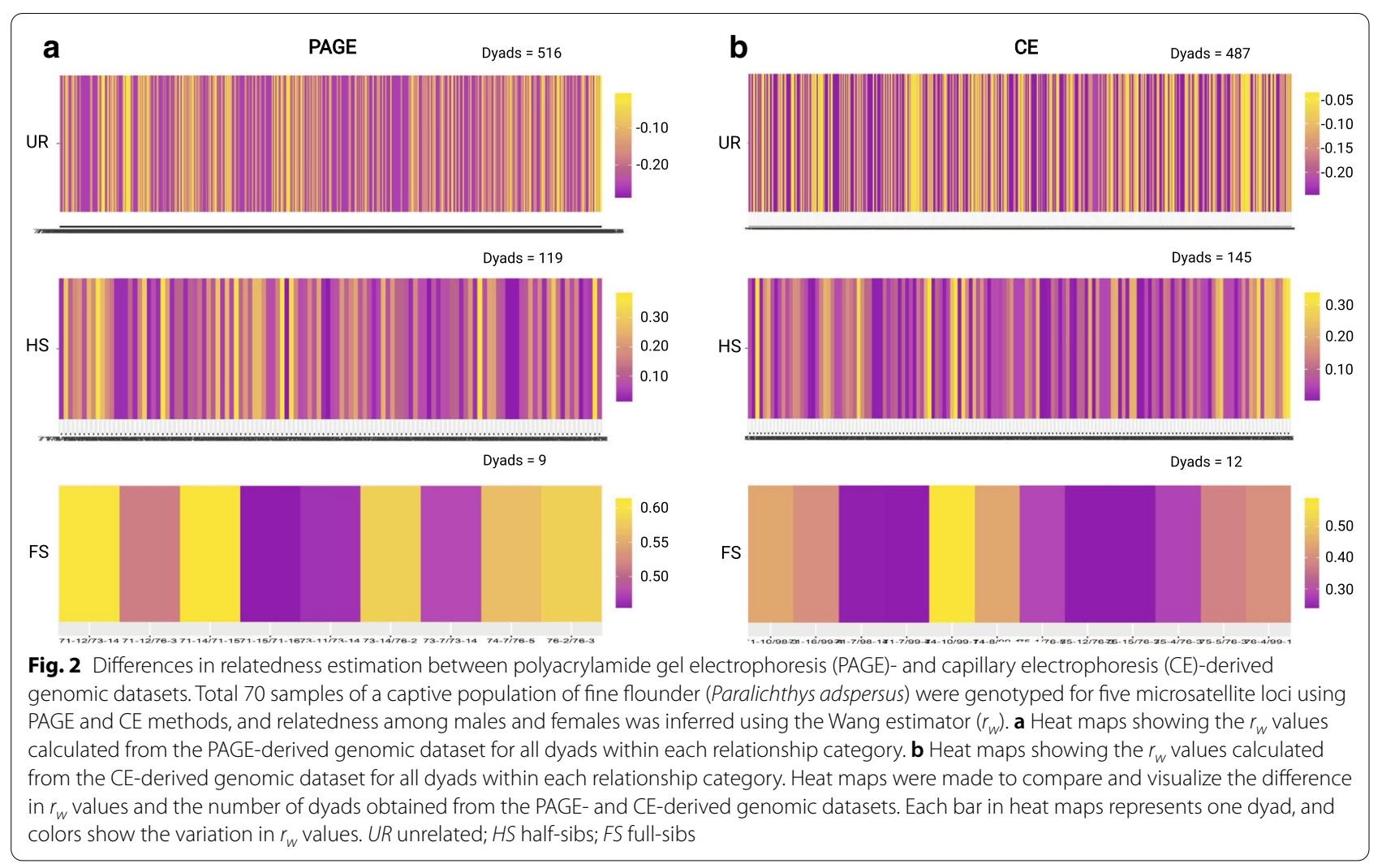

and labor-intensive, ultimately allow the visualization of one-base differences.

Although the discrepancies in allele frequency between PAGE- and CE-derived genomic datasets did not affect genetic diversity estimation, it is considerable enough to bear in mind that they did affect more complex analyses such as kinship assignation, in which a single erroneously identified allele leads to misinterpretations [20, 21]. Indeed, we obtained different patterns of relatedness while inferring relatedness from each dataset. Many studies have shown that when the genomic data are teeming with mistyping errors, the actual relatedness among individuals is difficult to detect, as error rates as low as 0.01 per allele can result in a rate of false paternity exclusion exceeding 20\% [9, 11, 12]. An alternative explanation for the disagreement between both datasets in terms of relatedness is the presence of low heterozygosity in the captive population (less than $50 \%$ on average), which can prevent precise parentage assignation unless a large number of microsatellite loci are combined with a nearcomplete sampling of the parental generation [42]. If the analysis of more loci through the PAGE method can lead to the correct categorization of dyads, however, requires further investigation. Nevertheless, as parentage testing is a powerful tool to study life history and establish sustainable breeding programs if estimated accurately [42], our results showed that researchers should not rely on PAGE-derived genomic data to infer relatedness; instead, they should utilize genomic data generated from HTP methods, which can accomplish allele sizing with higher resolution and greater accuracy [20].

\section{Conclusion}

Typographical errors within the PAGE-derived genomic data led to discrepancies in relatedness patterns; nonetheless, the PAGE-and CE-derived genomic datasets gave the same conclusions regarding genetic diversity. Therefore, such findings suggest that the PAGE system is an efficient and less costly alternative to the HTP methods in a population research framework, with the advantage that any laboratory with minimum infrastructure can accomplish it.

\section{Limitations}

While the evidence we have shown provides strong support for the effect of the genotyping technique in kinship assignment, our study is not without certain caveats. To address some of them, we should mention that the accuracy of relatedness estimation improves as the number of loci increases [43]. For instance, while the proportion of successfully identified parent-offspring dyads can be up to 0.75 if five microsatellite loci are used, this proportion 
increases to 0.95 if 20 microsatellites are used [43]. Moreover, it has been shown that genotyping with less than 17 microsatellite loci leads to significant deviations in population genetic results by affecting the stability of genetic distance parameters [3, 44, 45]. Because an increasing number of microsatellite loci could have affected the results of both PAGE- and CE-derived genomic datasets, the assessment of this variable together with the technique used warrants further investigation. Finally, this study considers relatedness analysis based on the empirical kinship coefficient alone estimated using genomic data since our dataset did not include pedigree information. Therefore, further study on the precision of the $\mathrm{CE}$ method in estimating true relatedness is needed.

\section{Abbreviations}

A: Number of alleles; $\mathrm{a}_{\mathrm{e}}$ : Effective number of alleles; APS: Ammonium persulfate; CE: Capillary electrophoresis; FS: Full-sibs; He: Expected heterozygosity; Ho: Observed heterozygosity; HS: Half-sibs; PAGE: Polyacrylamide gel electrophoresis; PIC: Polymorphic information content; R: Allelic richness; $r_{w}$ : Wang estimator; $r_{x y}$ : Coefficient of relatedness; TEMED: Tetramethylethylenediamine; UR: Unrelated; Allele: One of the possible forms of a gene at a given locus; Allelic dropout: Source of missing data in which one or both allelic copies at a locus fail to be amplified by PCR; Allele frequency: Relative proportion of an allele within a population; Allelic richness: Number of alleles in a set of populations determined taking into account the smallest population size; Genetic relatedness: The probability that two individuals share an allele due to recent common ancestry; Heterozygosity: The proportion of individuals within a population that carry two different alleles at a locus; Microsatellite hypervariability: Accumulation of length mutations during replication at a given micros atellite locus; Null alleles: Amplification failure due to mutations in the binding site of at least one primer; PIC: Discriminatory power of loci considering allele number and relative frequency; Stuttering: Series of minor peaks immediately adjacent to the major peak for a given locus in a gel.

\section{Supplementary Information}

The online version contains supplementary material available at https://doi. org/10.1186/s13104-021-05861-9.

\section{Additional file 1: Figure S1. Capillary electrophoresis (CE)- and} polyacrylamide gel electrophoresis (PAGE)-derived genomic datasets give comparable genetic diversity measures. Total 40 samples of a wild population of fine flounder (Paralichthys adspersus) were genotyped for five microsatellite loci using CE and PAGE methods, and the genetic diversity levels obtained from both genomic datasets were compared. a-f Violin plots with included boxplots summarizing the data distribution of different genetic diversity parameters. $\mathbf{g}$ Violin plots with included boxplots summarizing the distribution of null allele frequencies. Each box plot shows the median (middle line) and interquartile range (boxes). The bottom and top of each box indicate the 25th and 75th percentiles, while whiskers represent the minimum and maximum. $\mathbf{h}$ Allele frequency spectra of the five microsatellite loci used. Statistical analysis was performed using unpaired t tests. PIC, polymorphic information content; $A$ number of alleles; $a_{e}$ effective number of alleles; $R$ allelic richness; Ho observed heterozygosity; He expected heterozygosity. Figure S2. Differences in relatedness estimation between capillary electrophoresis (CE)- and polyacrylamide gel electrophoresis (PAGE)-derived genomic datasets. Total 40 samples of a wild population of fine flounder (Paralichthys adspersus) were genotyped for five microsatellite loci using CE and PAGE methods, and relatedness among males and females was inferred using the Wang estimator $\left(r_{w}\right)$. a Heat maps showing the $r_{w}$ values calculated from the CEderived genomic dataset for all dyads within each relationship category. b Heat maps showing the $r_{w}$ values calculated from the PAGE-derived genomic dataset for all dyads within each relationship category. Heat maps were made to compare and visualize the difference in $r_{w}$ values and the number of dyads obtained from the CE- and PAGE-derived genomic datasets. Each bar in heat maps represents one dyad, and colors show the variation in $r_{w}$ values. UR unrelated; HS half-sibs; FS full-sibs. Table S1. PCR conditions and size range of the fragments for each microsatellite locus. Table S2. Genetic diversity obtained from capillary electrophoresis (CE)and polyacrylamide gel electrophoresis (PAGE)-derived genomic data for a captive population of Paralichthys adspersus. Repeat motif, annealing temperature (Ta), frequency of null alleles, polymorphic information content (PIC), number of alleles per locus (A), effective number of alleles $\left(a_{e}\right)$, allelic richness (R), and observed and expected heterozygosity ( $\mathrm{Ho} / \mathrm{He})$ for each microsatellite locus are shown. Table S3. Genetic diversity obtained from capillary electrophoresis (CE)- and polyacrylamide gel electrophoresis (PAGE)-derived genomic data for a wild population of Paralichthys adspersus. Repeat motif, annealing temperature (Ta), frequency of null alleles, polymorphic information content (PIC), number of alleles per locus (A), effective number of alleles $\left(a_{e}\right)$, allelic richness $(R)$, and observed and expected heterozygosity $(\mathrm{Ho} / \mathrm{He})$ for each microsatellite locus are shown.

\section{Acknowledgements}

We want to thank the personnel at Pacific Deep-Frozen S.A. for providing us all the help and facilities for the correct sampling of Paralichthys adspersus.

\section{Authors' contributions}

All authors have contributed to the article preparation and finalization. Detailed author contribution are as follows: JSV: methodology, investigation, formal analysis, data curation, validation, writing —original draft preparation, writing-review and editing. LRF: supervision, methodology, formal analysis, data curation, validation, writing - review and editing. CYB: conceptualization, visualization, writing-review and editing, project administration. EZM: conceptualization, writing - review and editing, project administration. All authors read and approved the final manuscript.

\section{Funding}

This research did not receive any specific Grant from funding agencies in the public, commercial, or not-for-profit sectors.

\section{Availability of data and materials}

The datasets used and/or analyzed during the current study are available from the corresponding author on reasonable request.

\section{Declarations}

\section{Ethics approval and consent to participate}

Not applicable.

\section{Consent for publication}

Not applicable.

\section{Competing interests}

The authors declare that they have no competing interests.

Received: 17 June 2021 Accepted: 26 November 2021

Published online: 07 December 2021

\section{References}

1. Spritz RA. Duplication/deletion polymorphism $5^{\prime}$ - to the human $\beta$ globin gene. Nucleic Acids Res. 1981;9:5037-48. https://doi.org/10.1093/ nar/9.19.5037.

2. Ellegren H. Microsatellites: simple sequences with complex evolution. Nat Rev Genet. 2004;5:435-45. https://doi.org/10.1038/nrg1348.

3. Wang $\mathrm{H}$, Yang $\mathrm{B}$, Wang $\mathrm{H}$, Xiao $\mathrm{H}$. Impact of different numbers of microsatellite markers on population genetic results using SLAF-seq data for Rhododendron species. Sci Rep. 2021;11:8597. https://doi.org/10.1038/ s41598-021-87945-x. 
4. Pesso-Coelho NH, Vagner-Tambarussi E, Ibanes-Aguiar B, Roque RH, Marcelo-Portela R, Chaves Braga R, et al. Understanding genetic diversity, spatial genetic structure, and mating system through microsatellite markers for the conservation and sustainable use of Acrocomia aculeata (Jacq.) Lodd. Ex Mart. Conserv Genet. 2018;19:879-91. https:// doi.org/10.1007/s10592-018-1061-Z.

5. Yıldırım Y, Anderson MJ, Hansson B, Patel S, Millar CD, Rainey PB. Genetic structure of the grey side-gilled sea slug (Pleurobranchaea maculata) in coastal waters of New Zealand. PLoS ONE. 2018;13: e0202197. https://doi.org/10.1371/journal.pone.0202197.

6. Nordahl O, Koch-Schmidt P, Sunde J, Yıldırım Y, Tibblin P, Forsman A, et al. Genetic differentiation between and within ecotypes of pike (Esox lucius) in the Baltic Sea. Aquat Conserv Mar Freshw Ecosyst. 2019;29:1923-35. https://doi.org/10.1002/aqc.3196.

7. Ohashi J, Tokunaga K. Power of genome-wide linkage disequilibrium testing by using microsatellite markers. J Hum Genet. 2003;48:487-91. https://doi.org/10.1007/s10038-003-0058-7.

8. Schlötterer C. Hitchhiking mapping - functional genomics from the population genetics perspective. Trends Genet. 2003;19:32-8. https:// doi.org/10.1016/s0168-9525(02)00012-4.

9. Dakin EE, Avise JC. Microsatellite null alleles in parentage analysis. Heredity. 2004;93:504-9. https://doi.org/10.1038/sj.hdy.6800545.

10. Miller CR, Joyce P, Waits LP. Assessing allelic dropout and genotype reliability using maximum likelihood. Genetics. 2002;160:357-66.

11. Wang J. Estimating genotyping errors from genotype and reconstructed pedigree data. Methods Ecol Evol. 2018;9:109-20. https://doi. org/10.1111/2041-210X.12859.

12. Hoffman Jl, Amos W. Microsatellite genotyping errors: detection approaches, common sources and consequences for paternal exclusion. Mol Ecol. 2005;14:599-612. https://doi.org/10.1111/j.1365-294X. 2004.02419.x

13. Dewoody J, Nason JD, Hipkins VD. Mitigating scoring errors in microsatellite data from wild populations. Mol Ecol Notes. 2006;6:951-7. https://doi.org/10.1111/j.1471-8286.2006.01449.x.

14. Kumar M, Kim SR, Sharma PC, Pareek A. Simple and efficient way to detect small polymorphic bands in plants. Genomics Data. 2015;5:21822. https://doi.org/10.1016/j.gdata.2015.06.006.

15. Kirschner $P$, Arthofer W, Pfeifenberger S, Záveská E, Schönswetter $P$, Frajman $B$, et al. Performance comparison of two reduced-representation based genome-wide marker-discovery strategies in a multi-taxon phylogeographic framework. Sci Rep. 2021;11:3978. https://doi.org/10. 1038/s41598-020-79778-x.

16. Buel E, Schwartz MB, LaFountain MJ. Capillary electrophoresis STR analysis: comparison to gel-based systems. J Forensic Sci. 1998;43:164-70.

17. Al Shaye N, Migdadi H, Charbaji A, Alsayegh S, Daoud S, AL-Anazi W, et al. Genetic variation among Saudi tomato (Solanum lycopersicum L.) landraces studied using SDS-PAGE and SRAP markers. Saudi J Biol Sci. 2018;25:1007-15. https://doi.org/10.1016/j.sjbs.2018.04.014.

18. Shi Y, Yu L, Han X, Zhao S, Niu T, Xu C. Development of 12 microsatellite markers for Bombina orientails based on RNA-Seq and their usefulness in population genetic diversity. Mol Biol Rep. 2018;45:2811-4. https:// doi.org/10.1007/s11033-018-4371-9.

19. Hamouda M. Molecular analysis of genetic diversity in population of Silybum marianum (L.) Gaertn in Egypt. J Genet Eng Biotechnol. 2019:17:1-9. https://doi.org/10.1186/s43141-019-0011-6.

20. Vemireddy LR, Archak S, Nagaraju J. Capillary electrophoresis is essential for microsatellite marker based detection and quantification of adulteration of Basmati rice (Oryza sativa). J Agric Food Chem. 2007;55:8112-7. https://doi.org/10.1021/jf0714517.

21. Pagel UR, Reis RS, Carvalho VP, Santos EVW, Zandonade E, Louro ID, et al. Comparative analysis of short tandem repeat data obtained by automated and gel electrophoresis techniques. Genet Mol Res. 2016 https://doi.org/10.4238/gmr.15038436.

22. Stewart S, Wickramasinghe D, Dorrance AE, Robertson AE. Comparison of three microsatellite analysis methods for detecting genetic diversity in Phytophthora sojae (Stramenopila: Oomycete). Biotechnol Lett. 2011;33:2217-23. https://doi.org/10.1007/s10529-011-0682-9.

23. Coimbra MRM, Hasegawa O, Kobayashi K, Koretsugu S, Ohara E, Okamoto N. Twenty microsatellite markers from the Japanese flounder Paralichthys olivaceus. Fish Sci. 2001;67:358-60. https://doi.org/10. 1046/j.1444-2906.2001.00215.x.
24. Sekino M, Hara M. Application of microsatellite markers to population genetics studies of Japanese flounder Paralichthys olivaceus. Mar Biotechnol. 2001;3:572-89. https://doi.org/10.1007/s10126-001-0064-8.

25. Kim WJ, Kang JH, Nam BH, Kong HJ, Park EM, Lee JH, et al. Development of 52 new polymorphic microsatellite markers for the olive flounder, Paralichthys olivaceus. Mol Ecol Resour. 2009;9:839-42. https://doi. org/10.1111/j.1755-0998.2009.02287.x.

26. Taggart JB, Hynes RA, Prodöuhl PA, Ferguson A. A simplified protocol for routine total DNA isolation from salmonid fishes. J Fish Biol. 1992;40:963-5. https://doi.org/10.1111/j.1095-8649.1992.tb02641.x.

27. Gallagher SR. One-dimensional SDS gel electrophoresis of proteins. Curr Protoc Mol Biol. 2006;75:10.2.1-10.2A.37. https://doi.org/10.1002/ 0471142727.mb1002as75.

28. Rangel-Villalobos H, Salazar-Flores J, Muñoz-Valle JF, Torres-Jasso JH, Páez-Riberos LA. Alternative silver stain detection protocol for the GenePrint ${ }^{\circledR}$ STR system applied to mestizo population from Jalisco (West of Mexico). J Forensic Res. 2010;1:105. https://doi.org/10.4172/ 2157-7145.1000105

29. Van Oosterhout C, Hutchinson WF, Wills DPM, Shipley P. MICROCHECKER: software for identifying and correcting genotyping errors in microsatellite data. Mol Ecol Notes. 2004;4:535-8. https://doi.org/10. 1111/j.1471-8286.2004.00684.x.

30. Kalinowski ST, Taper ML. Maximum likelihood estimation of the frequency of null alleles at microsatellite loci. Conserv Genet. 2006;7:9915. https://doi.org/10.1007/s10592-006-9134-9.

31. Kalinowski ST, Taper ML, Marshall TC. Revising how the computer program CERVUS accommodates genotyping error increases success in paternity assignment. Mol Ecol. 2007;16:1099-106. https://doi.org/ 10.1111/j.1365-294X.2007.03089.x.

32. Peakall R, Smouse PE. GenALEx 6.5: genetic analysis in Excel. Population genetic software for teaching and research-an update. Bioinformatics. 2012;28:2537-9. https://doi.org/10.1093/bioinformatics/bts460.

33. Kalinowski ST. HP-RARE 1.0: a computer program for performing rarefaction on measures of allelic richness. Mol Ecol Notes. 2005;5:187-9. https://doi.org/10.1111/j.1471-8286.2004.00845.x.

34. Allaire JJ. RStudio: integrated development environment for R. https:// www.r-project.org/. Accessed 1 Jun 2021.

35. Wang J. An estimator for pairwise relatedness using molecular markers. Genetics. 2002;160:1203-15.

36. Wang J. Coancestry: a program for simulating, estimating and analysing relatedness and inbreeding coefficients. Mol Ecol Resour. 2011:11:141-5. https://doi.org/10.1111/j.1755-0998.2010.02885.x.

37. Blouin MS, Parsons M, Lacaille V, Lotz S. Use of microsatellite loci to classify individuals by relatedness. Mol Ecol. 1996;5:393-401. https:// doi.org/10.1111/j.1365-294X.1996.tb00329.x

38. Sánchez-Velásquez JJ, Pinedo-Bernal PN, Reyes-Flores LE, YzásigaBarrera C, Zelada-Mázmela E. Genetic diversity and relatedness inferred from microsatellite loci as a tool for broodstock management of fine flounder Paralichthys adspersus. Aquac Fish. 2021. https://doi.org/10. 1016/J.AAF.2021.06.008.

39. Wang J. Marker-based estimates of relatedness and inbreeding coefficients: an assessment of current methods. J Evol Biol. 2014;27:518-30. https://doi.org/10.1111/jeb.12315.

40. Wang J. Estimating pairwise relatedness in a small sample of individuals. Heredity. 2017;119:302-13. https://doi.org/10.1038/hdy.2017.52.

41. Wright S. Systems of mating. V. General considerations. Genetics. 1921;6:167-78. https://doi.org/10.1093/genetics/6.2.167.

42. Tokarska M, Marshall T, Kowalczyk R, Wójcik JM, Pertoldi C, Kristensen TN, et al. Effectiveness of microsatellite and SNP markers for parentage and identity analysis in species with low genetic diversity: the case of European bison. Heredity. 2009;103:326-32. https://doi.org/10.1038/ hdy.2009.73

43. Wagner AP, Creel S, Kalinowski ST. Estimating relatedness and relationships using microsatellite loci with null alleles. Heredity. 2006;97:33645. https://doi.org/10.1038/sj.hdy.6800865.

44. Reiner $\mathrm{G}$, Lang M, Willems $\mathrm{H}$. Impact of different panels of microsatellite loci, different numbers of loci, sample sizes, and gender ratios on population genetic results in red deer. Eur J Wildl Res. 2019;65:1-12. https://doi.org/10.1007/S10344-019-1262-X.

45. Koskinen MT, Hirvonen H, Landry PA, Primmer CR. The benefits of increasing the number of microsatellites utilized in genetic population 
studies: an empirical perspective. Hereditas. 2004;141:61-7. https://doi. org/10.1111/J.1601-5223.2004.01804.X

\section{Publisher's Note}

Springer Nature remains neutral with regard to jurisdictional claims in published maps and institutional affiliations.

Ready to submit your research? Choose BMC and benefit from:

- fast, convenient online submission

- thorough peer review by experienced researchers in your field

- rapid publication on acceptance

- support for research data, including large and complex data types

- gold Open Access which fosters wider collaboration and increased citations

- maximum visibility for your research: over 100M website views per year

At BMC, research is always in progress.

Learn more biomedcentral.com/submissions 